University of Nebraska - Lincoln

DigitalCommons@University of Nebraska - Lincoln

9-1-1996

\title{
High coercive Field and Film Stress for epitaxial Monolayers of Fe on $\mathrm{W}(110)$
}

D. Sander

Max-Planck-Institut fur Mikrostrukturphysik, Weinberg 2,06120 Halle, Germany

Axel Enders

Max-Planck-Institut fur Mikrostrukturphysik, a.enders@me.com

Ralph Skomski

University of Nebraska-Lincoln, rskomski2@unl.edu

J. Kirschner

Max-Planck-Institut fur Mikrostrukturphysik, Weinberg 2,06120 Halle, Germany

Follow this and additional works at: https://digitalcommons.unl.edu/physicsskomski

Part of the Physics Commons

Sander, D.; Enders, Axel; Skomski, Ralph; and Kirschner, J., "High coercive Field and Film Stress for epitaxial Monolayers of Fe on W(110)" (1996). Ralph Skomski Publications. 9.

https://digitalcommons.unl.edu/physicsskomski/9

This Article is brought to you for free and open access by the Research Papers in Physics and Astronomy at DigitalCommons@University of Nebraska - Lincoln. It has been accepted for inclusion in Ralph Skomski Publications by an authorized administrator of DigitalCommons@University of Nebraska - Lincoln. 


\title{
High coercive Field and Film Stress for epitaxial Monolayers of Fe on W(110)
}

\author{
D. Sander, A. Enders, R. Skomski, and J. Kirschner
}

Max-Planck-Institut für Mikrostrukturphysik, Weinberg 2, 06120 Halle, Germany

\begin{abstract}
Elastic and magnetic properties of ultrathin iron films on tungsten(110) are investigated. In situ film stress measurements during growth show stress values of $25 \mathrm{GPa}$ per deposited monolayer. Our experiments indicate that the tremendous film stress triggers the formation of a misfit dislocation network at a coverage of 1.5 monolayers. The effect of the film stress and its spatial variation on the magnetic behavior are discussed. We find an high coercivity of order $0.3 \mathrm{~T}$ for 1.5 monolayer thick films. A model is presented that explains the high coercivity in terms of strong domain wall pinning.
\end{abstract}

\section{INTRODUCTION}

Recent experiments on the magnetism of monolayer films of iron on tungsten have revealed interesting magnetic properties like the finite width scaling of the Curie temperature of iron stripes [1], or the reportedly [2] surpressed long range magnetic order in a narrow iron thickness range below 1.5 monolayers. The influence of film morphology on magnetism was discussed by Elmers et al. [1] in terms of the correlation between the percolation of iron islands and the onset of ferromagnetic order in the film, both found to occur at a coverage of 0.6 monolayers. The goal of this paper is to contribute additional aspects of the correlation between film morphology and magnetism by combining Kerr-effect measurements with in situ film stress measurements during growth. Thus, hysteresis loops can be closely related to certain stress states in the film. Most interestingly, at the film thickness where we expect the formation of a misfit distortion network from our stress vs. coverage curves, we observe an extremely increased coercivity. We discuss the high coercivity of order $0.3 \mathrm{~T}$ in terms of strong domain wall pinning. An estimate for the stress induced contribution to the coercivity is given.

\section{EXPERIMENTAL}

We performed the stress and magneto-optic Kerr-effect (MOKE) measurements [3] in a two level ultra high vacuum (UHV) chamber shown in Fig. 1. The sample and the films are prepared in the top level. Here, film stress is measured during growth using a simple bending beam technique described earlier [4]. The sample can be lowered to the second level, where the magnetic properties of the grown film are characterized by MOKE. The essential experimental detail of this set-up is the strong electromagnet with the coils located under the vacuum chamber, and with an yoke penetrating the chamber walls (Fig. 1). Fields of up to $0.3 \mathrm{~T}$ can be produced at the sample location. As will be discussed later, such high fields

Manuscript received February 29, 1996.

D. Sander, e-mail: sander@mpi-msp-halle.mpg.de fax 49-345-5511-223.

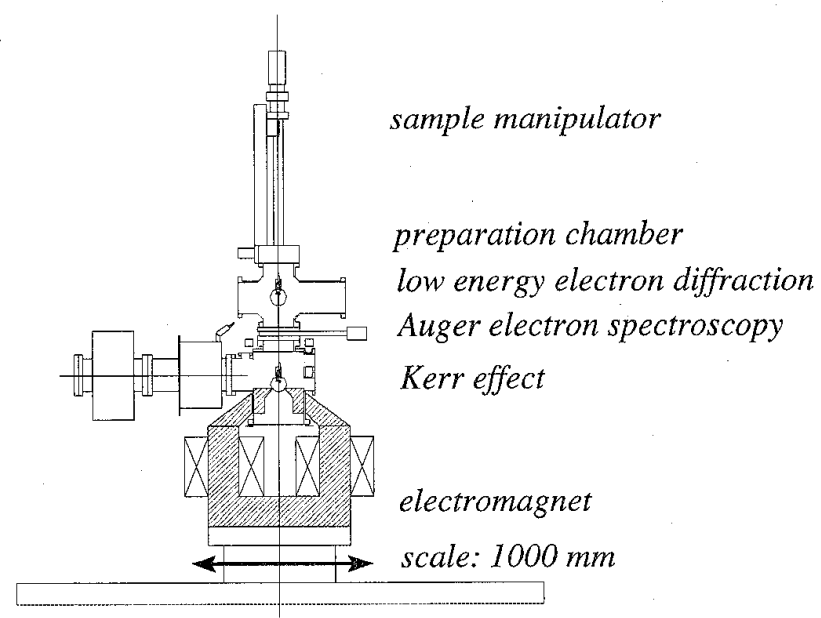

Fig. 1. UHV-chamber with electromagnet.

in conjunction with sample cooling capabilities can be crucial to properly investigate monolayer magnetism.

\section{FILM STRESS AND MAGGNETISM}

The heteroepitaxy of iron on tungsten is governed mainly by the large lattice mismatch $f$ between the two elements. From the lattice constants of iron $\left(\mathrm{a}_{\mathrm{Fe}}=2.866 \AA\right)$ and tungsten $\left(\mathrm{a}_{\mathrm{w}}=3.165 \AA\right)$, we calculate $f=\left(\mathrm{a}_{\mathrm{W}}-\mathrm{a}_{\mathrm{Fe}}\right) / \mathrm{a}_{\mathrm{w}}=0.094$. In spite of this mismatch of $9.4 \%$ it is known from a number of experiments [5][6], that the first layer of iron grows pseudomorphically on W(110). As a result of this pseudomorphic growth, the iron layer is heavily strained to match the atomic distances of the tungsten substrate. Thus, it is expected from elastic theory [7], that from a so called critical film thickness on, the growth mode of the iron film changes. At the critical film thickness, the increasing strain energy drives the formation of misfit dislocations in the growing film. Recent scanning tunneling microscopy (STM) experiments [6] indicate, that even for an iron deposition of less than 2 pseudomorphic monolayers [8], the formation of a distortion network starts.

In Fig. 2 we present stress measurements taken during the growth of 25 monolayers $(40 \AA)$ of $\mathrm{Fe}$ on $\mathrm{W}(110)$ at $300 \mathrm{~K}$. The deposition of up to 0.5 monolayers of iron induces compressive film stress, which is opposite in sign for what is expected for a strained iron film. With continuing deposition, tensile film stress sets in, and at 1.5 monolayers a kink in the stress vs. coverage curve is observed. The slope of the curve decreases by a factor of two from $4 \mathrm{~N} / \mathrm{m}$ to $2 \mathrm{~N} / \mathrm{m}$ per deposited monolayer. Interrupting the growth at any point, freezes the stress at the respective value of the stress curve. 


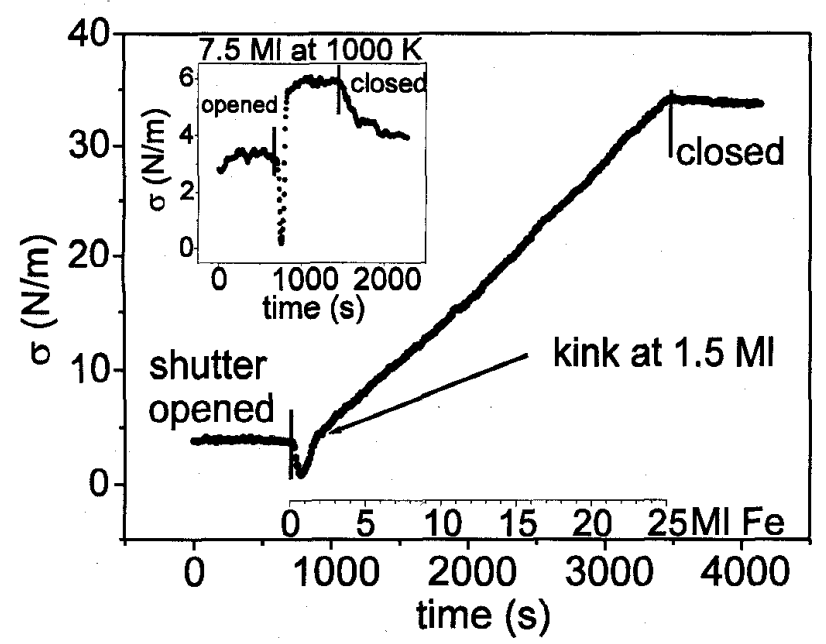

Fig. 2. Stress measurements taken during the growth of 25 monolayers of iron at $300 \mathrm{~K}$. The kink indicates the formation of a dislocation network in the film. The inset shows the stress evolution during the deposition of 7.5 monolayers at $1000 \mathrm{~K}$. The deposition was calibrated using a quartz oscillator.

Note that the film stress is of order $4 \mathrm{~N} / \mathrm{m}$ per monolayer $(25 \mathrm{GPa})$. This is an amazingly high stress value, far above the elasticity limits of high strength materials.

We assign the compressive stress for coverages below 0.5 monolayers to a mere surface stress effect. From STM experiments [1] it is known that in this coverage range seperate islands of $\mathrm{Fe}$-atoms nucleate on the substrate, and these islands do not percolate up to a coverage of 0.6 monolayers. Therefore, the picture of a strained layer is certainly questionable for thicknesses below percolation. Below percolation, we are rather dealing with adsorbate induced surface stress, caused by the electronic interaction of the Fe-adsorbate with the W-substrate. The details of this interaction are beyond the scope of this contribution. We refer to earlier experiments [9] that have shown considerable compressive surface stress induced by adsorption processes.

We correlate the kink in the stress curve with the onset of the formation of the distortion network at a coverage of 1.5 monolayers. This reasoning is supported by our additional STM experiments that will be published elsewhere [10] and by [6]. We conclude that the film is driven by the increasing stress to introduce misfit dislocations once a film stress of approximately $25 \mathrm{GPa}$ is reached. At the low growth temperature of $300 \mathrm{~K}$, the stress relief due to the distortion network is not complete. The constant slope of our stress curve above the kink indicates a constant stress in the film. The stress relief mechanism due to the introduction of misfit dislocations at the interface between the first and the second layer of iron is more effective only at higher temperatures. Performing the experiments at $1000 \mathrm{~K}$ (see inset Fig. 2), leaves only the first monolayer strained, whereas the deposition of iron in excess of the first layer induces no additional stress in the film. As shown in the inset of Fig. 2, after closing of the shutter, the stress relaxes to the monolayer value of $4 \mathrm{~N} / \mathrm{m}$.

The kink of the stress vs. coverage curve does not only (a)

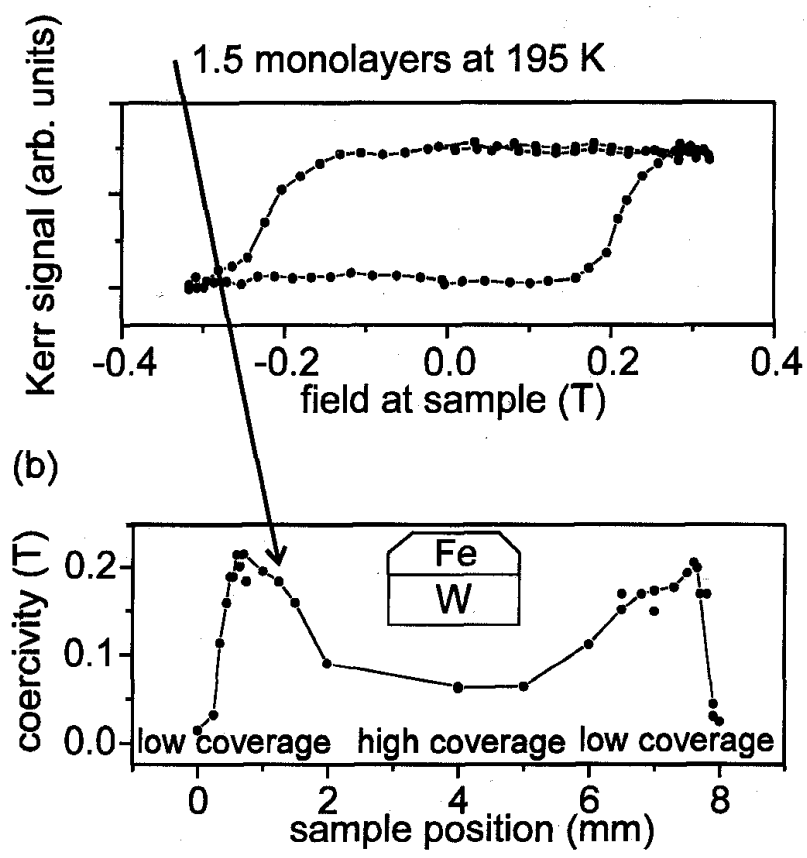

Fig. 3. (a) Hysteresis loop of 1.5 monolayers taken at $195 \mathrm{~K}$. At $140 \mathrm{~K}$, the coercivity is higher than $0.3 \mathrm{~T}$. (b) Thickness dependence of the coercivity measured on an iron film with a mesa-shaped thickness profile - see text -. The arrow points to the coverage of 1.5 monolayers on the mesa-shaped film.

indicate some structural change in the film. At that coverage of 1.5 monolayers also an unusual high coercivity larger than $0.3 \mathrm{~T}$ is observed. Figure 3(a) shows the hysteresis loop of a 1.5 monolayer film. Note, that the coercivity is $0.2 \mathrm{~T}$ at $195 \mathrm{~K}$, at $140 \mathrm{~K}$ it is above our field limit of $0.3 \mathrm{~T}$. To examine the coverage dependence of the coercivity, we measured the coercivity on a Fe-film with a mesa-like thickness variation (Fig. 3(b)): The film thickness increases from 0.8 monolayers at one end of the sample up to 2 monolayers on the plateau and decreases down to 0.8 monolayers at the other end of the

(a)

(b)

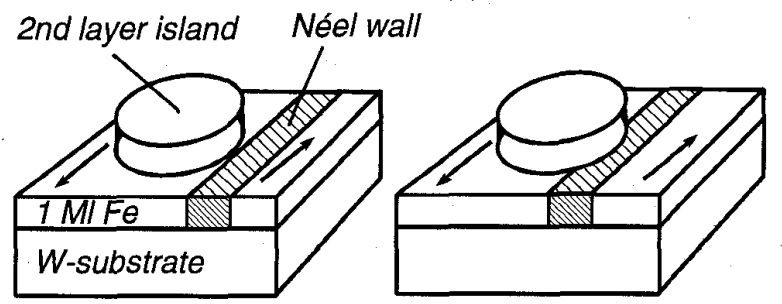

Fig. 4. (a) A Néel wall moves in the monolayer region, seperating domains of opposite magnetization. (b) The domain movement is pinned at a second layer island due to the increased domain wall energy in that island. The arrows indicate the direction of magnetization. 
sample. A clear maximum of the coercivity in a coverage range of $1.3 \pm 0.2$ monolayers is obvious from Fig. 3 (b).

In the following we propose a model for the high coercivity in terms of strong domain wall pinning. Generally, nucleation of domains with reversed magnetization and pinning of domain walls are considered to be the main coercivity mechanisms [11][12]. However, the low coercivity of one and two monolayer thick films (Fig. 3(b)) indicates the presence of nucleation centres, leaving domain wall pinning as the main coercivity mechanism. The main input for our model comes from the dependence of the domain wall energy on the iron film thickness: A domain wall moves in the monolayer region till it hits a second layer patch of Fe-atoms (Fig. 4(a)). The increased number of iron-iron bonds in the second layer patch in comparison to the surrounding one monolayer region leads to an increased exchange energy of the wall when the wall enters the second layer patch. Thus, it is energetically unfavorable for the wall to enter the two monolayer region due to the increased wall energy. As a result, the domain wall is pinned at the second layer patch (Fig. 4(b)). The size of the second layer islands can be extracted from STM pictures: At a coverage of 1.5 monolayers the patches are approximately $10 \mathrm{~nm}$ wide and $50 \mathrm{~nm}$ long [6] [10]. We take the value for the in-plane surface anisotropy of Fe on W(110) given in [13] to estimate the width of the Néel wall to be of order $5 \mathrm{~nm}$ [14]. Thus, we conclude that the second layer islands are suitable for strong domain wall pinning.

Another contribution to the increased coercivity originates from the inhomogenous stress field in the iron film once misfit dislocations are formed at a coverage of 1.5 monolayers. At the beginning of the formation of the misfit dislocation network, the spatial variation of the stress is at a maximum. Bethge $e$ al. [6] have shown, that the formation of a distortion network starts at a coverage of 1.5 monolayers at the interface between second and first layer islands. This means that the homogenous stress field of the pseudomorphic monolayer is disturbed at the second layer patches. Usually the resulting coercivity contribution is considered to be pretty small, but our stress measurements indicate that the stress is lowered by $50 \%$ due to the misfit dislocations. This results in a tremendous stress variation of $12 \mathrm{GPa}$ over distances of order $20 \mathrm{~nm}$. Following an argumentation given in Ref. [11], a coercivity contribution of $70 \mathrm{mT}$ can be estimated. Thus, we assign the major contribution to the high coercivity of the 1.5 monolayer film to a strong domain wall pinning.

\section{CONCLUSION}

We have shown that in situ film stress measurements during growth give important additional quantitative insight in growth phenomena like the formation of misfit dislocations. The measured stress values indicate that a stress induced contribution to coercivity is not negligible in general due to the tremendous stress values found for monolayer films. The major contribution to coercivity originates however from a strong domain wall pinning at two monolayer patches, where the exchange energy of the wall is higher than in the surrounding one monolayer region.

\section{REFERENCES}

[1] H.J. Elmers, J. Hausschild, H. Höche, U. Gradmann, H. Bethge, D. Heuer, and U. Köhler, Phys. Rev. Lett. 73, 898 (1994).

[2] H.J. Elmers, J. Hausschild, H. Fritzsche, G. Liu, U. Gradmann, and U. Köhler, Phys. Rev. Lett. 75, 2031 (1995).

[3] S. D. Bader, J. Magn. Mater. 100, 440 (1991).

[4] D. Sander, A. Enders, and J. Kirschner, Rev. Sci. Instrum. 66, 4734 (1995).

[5] U. Gradmann and G. Waller, Surf. Sci. 116, 539 (1982).

[6] H. Bethge; D. Heuer, Ch. Jensen, K. Reshöft, and U. Köhler, Surf. Sci. 331-333, 878 (1995)

[7] Jan H.van der Merwe, J. Woltersdorf, and W. A. Jesser, Mat. Sci. and Engineer. 81, 1 (1986)

[8] One pseudomorphic monolayer $\mathrm{Fe}$ is defined by $1.41 \cdot 10^{15} \mathrm{Fe}$-atoms $/ \mathrm{cm}^{2}$. This equals one $\mathrm{Fe}$-atom per W(110)-surface atom. Throughout this paper one monolayer (1 Ml) equals one pseudomorphic monolayer.

[9] D. Sander, U. Linke, and H. Ibach, Surf. Sci. 272, 318 (1992).

[10] D. Sander, R. Skomski, C. Schmidthals, A. Enders, and J. Kirschner, unpublished.

[11] E. Kneller, Ferromagnetismus, Springer, Berlin 1962.

[12] R. Skomski and J.M.D. Coey, Phys. Rev. B 48, 15812, (1993).

[13] H. Fritzsche, H.J. Elmers, and U. Gradmann, J. Magn. Magn. Mater. 135, 343 (1994).

[14] The wall width $\delta$ can be estimated to be $\delta=\pi \sqrt{\frac{A t}{2 K_{S, P}}}$. Taking the monolayer thickness $t=2,03 \AA$, the in-plane surface anisotropy $\mathrm{K}_{\mathrm{s}, \mathrm{P}}=0.5 \mathrm{~mJ} / \mathrm{m}^{2}[13]$, and the exchange stiffness $\mathrm{A}=10^{-11} \mathrm{~J} / \mathrm{m}$ gives $\delta=4.4 \mathrm{~nm}$. See the contribution by R. Skomski, D.Sander in this issue for a justification of this equation. 